\title{
Minimizing the Noise Penalty Due to Mutual Coupling for a Receiving Array
}

Karl F. Warnick

warnick@byu.edu

Leonid Belostotski

Peter Russer

Follow this and additional works at: https://scholarsarchive.byu.edu/facpub

Part of the Electrical and Computer Engineering Commons

\section{Original Publication Citation}

Karl F. Warnick, Bert Woestenburg, Leonid Belostotski,and Peter Russer. "Minimizing the Noise Penalty Due to Mutual Coupling for a Receiving Array," IEEE Transactions on Antennas and Propagation, Vol. 57, No. 6, June 2009.

\section{BYU ScholarsArchive Citation}

Warnick, Karl F.; Belostotski, Leonid; and Russer, Peter, "Minimizing the Noise Penalty Due to Mutual Coupling for a Receiving Array" (2008). Faculty Publications. 890.

https://scholarsarchive.byu.edu/facpub/890 


\title{
Minimizing the Noise Penalty Due to Mutual Coupling for a Receiving Array
}

\author{
Karl F. Warnick, Senior Member, IEEE, Leonid Belostotski, Member, IEEE, and Peter Russer, Fellow, \\ IEEE
}

\begin{abstract}
For phased array receivers, mutual coupling leads to beam-dependent effective impedances at array element ports. Front end amplifiers can be matched for optimal noise performance for one beam steering direction, but noise performance becomes poor at other steering directions. We analyze this noise penalty in terms of beam equivalent noise temperature for various amplifier noise matching conditions, and develop a new matching condition that minimizes the average beam equivalent receiver noise temperature over multiple beams. For nonbeamforming applications such as MIMO communications, we show that noise performance for coupled arrays can be quantified using the spectrum of an available receiver noise temperature correlation matrix.
\end{abstract}

\section{INTRODUCTION}

The performance of antenna arrays for multiple input multiple output (MIMO) communications, focal plane arrays for radio astronomy, direction finding, phased array radar, and other applications is influenced by mutual coupling. It has long been understood that coupling introduces limitations on element efficiency for transmitters [1] and reduces the signal diversity in multiantenna communication systems [2-4]. More recently, it has been shown that mutual coupling for receive arrays decreases the achievable system sensitivity through an increase in equivalent amplifier noise temperature [5]. Noise from front end amplifiers which load the antenna elements couples through the array and enters other receiver chains. Unless this noise coupling effect is taken into account in the matching condition between the array and amplifiers, the receiver noise increases and the achievable SNR is decreased [6].

In this paper, we study the noise penalty caused by mutual coupling for a phased array beamformer using the beam equivalent receiver noise temperature and the beam noise matching efficiency developed in [7, 8]. The beam equivalent receiver noise temperature is

K. F. Warnick is with the Department of Electrical and Computer Engineering, Brigham Young University, 459 Clyde Building, Provo, UT 84602 .

L. Belostotski is with the Department of Electrical and Computer Engineering, University of Calgary, 2500 University Drive, N.W., Calgary, Alberta, Canada.

P. Russer is with the Institut für Hochfrequenztechnik, Technische Universität München, Arcisstr. 21, D-80333 Munich, Germany. receiver noise power referred to an equivalent antenna temperature, and the noise matching efficiency is the ratio of the minimum equivalent noise temperature for one amplifier under optimal noise matching conditions to the beam equivalent receiver noise temperature (in this paper, we will neglect the small noise contribution from components of the receiver chain after the front end amplifiers). These definitions allow a generalization of classical single-amplifier noise matching concepts to array systems. As is the case with classical amplifier noise theory, minimizing equivalent noise temperature maximizes the system SNR and sensitivity.

For an uncoupled array, it is possible to match each amplifier to an array element in such a way that the equivalent amplifier noise temperature considering the combined noise signals from all the amplifiers is equal to the minimum noise temperature for one amplifier, regardless of how the signals are combined in beamforming. For a coupled array, the beam equivalent noise temperature is minimized over all possible beamformers if and only if the array is decoupled by a multiport matching network so that the mutual impedance matrix is diagonalized, and each decoupled port is optimally noise matched to its amplifier [6].

Multiport decoupling networks of this type have been proposed and studied to increase the signal diversity for MIMO array systems $[3,4,9]$. For some applications, such as ultrawideband communications and focal plane array feeds for radio astronomy, however, bandwidth requirements and stringent limitations on ohmic losses preclude the use of a decoupling network. A decoupling network requires lumped element or passive interconnections between all array element ports, leading to a complex physical structure. The decoupling network must be located before the front end amplifiers, so losses must be small to avoid an intolerable increase in thermal noise. For these reasons, it is important to consider suboptimal matching networks which require fewer interconnections between the array element ports.

These considerations motivate an open question in array noise matching. If the matching network between array elements and front end amplifiers is restricted to consist of simple two-port matching networks inserted between each element and front end amplifier, what 
is the optimal noise matching condition? Equivalently, how should the amplifier optimal source reflection coefficients $\left(\Gamma_{\mathrm{opt}}\right)$ or source impedances $\left(Z_{\mathrm{opt}}\right)$ be tuned to minimize noise for an array?

One possibility is the self impedance matching condition, for which the amplifiers are noise matched to the self impedances of the array elements. The impact on system performance of the self impedance matching condition relative to an optimal decoupling network has been studied for MIMO systems [5] and focal plane arrays [10]. The self-impedance match is convenient because it does not rely on assumptions about how the array outputs are processed and is not limited to beamforming arrays. For coupled arrays, however, the selfimpedance matching condition does not deliver optimal noise performance for any beam steering direction.

Improved noise performance for a beamforming array can be achieved by noise matching to active impedances or active reflection coefficients for a given beam steering direction. The use of the active impedance in determining the amplifier noise for a phased array was studied by Craeye et al. [11]. A particularly important result in the theory of noise matching for beamforming arrays was obtained by Woestenberg, who showed that for a given beamformer the amplifier noise is minimized by noise matching to the active reflection coefficient [12]. Maaskant and Woestenberg considered the active reflection coefficient for a focal plane array [13]. The active impedance noise matching condition yields an optimal beam equivalent receiver noise temperature and a noise matching efficiency of unity for a mutually coupled array, so that the noise penalty due to mutual coupling is eliminated.

With the active impedance matching condition, however, the noise matching efficiency is unity only for one particular beamformer. For a multi-beam system, beams which are different from the beamformer used to determine the active impedance match can have a much poorer beam equivalent receiver noise temperature. As a result, a different noise matching condition may have better overall performance over multiple steered beams. To find the best amplifier noise matching condition for a multi-beam array, we derive a matching condition that minimizes the average beam equivalent noise temperature over a range of beam steering directions.

To quantify noise performance for non-beamforming applications such as MIMO communications, we show that the beam equivalent noise temperature can be expressed in terms of an available receiver noise temperature correlation matrix, which is a natural array generalization of the classical equivalent receiver noise temperature for the single-channel case. The spectrum of this matrix defines the range of possible beam equivalent noise temperatures as the beam is steered. This matrix provides an intrinsic or beam-independent way to characterize the goodness of an amplifier noise matching condition.

Numerical simulations are used to compare the performance of the self impedance, active impedance, and minimum average beam equivalent noise temperature matching conditions for several example array cases. For these arrays, we also quantity noise performance in terms of the spectrum of the available receiver noise temperature correlation matrix.

All voltage and field quantities will be assumed to be phasors with time dependence relative to $e^{j \omega t}$. An overbar is used to denote three-dimensional field vectors, and vectors of voltages or currents at multiple ports are typeset in boldface.

\section{ARray Model}

A receiving array can be characterized as a Thévenin equivalent source network by the impedance matrix $\mathbf{Z}_{A}$ or scattering matrix $\mathbf{S}_{A}$ looking into the element ports together with the open circuit voltages induced at the element ports by a plane wave with a given polarization $\hat{p}$, electric field intensity $E_{0}$, and angle of incidence $\Omega$ $[14,15]$. The incident field is $\bar{E}(\mathbf{r})=\hat{p} E_{0} e^{-j \mathbf{k} \cdot \mathbf{r}}$, where $\hat{p}$ is a unit vector and the spherical angle of $\mathbf{k}$ is $-\Omega$. The voltage response $v_{o c, m}\left(\hat{p}, E_{0}, \Omega\right)$ is the embedded open circuit loaded receiving voltage pattern for the $m$ th array element. The open circuit voltages for the array can be represented as a column vector $\mathbf{v}_{o c}\left(\hat{p}, E_{0}, \Omega\right)$. By reciprocity, the open circuit loaded voltages are related to the fields radiated by the array when excited as a transmitter according to

$$
v_{\mathrm{oc}, m}\left(\hat{p}, E_{0}, \Omega\right)=\frac{4 \pi j r e^{j k r}}{\omega \mu I_{0}} E_{0} \hat{p} \cdot \bar{E}_{m}(\mathbf{r})
$$

where $\mathbf{r}=(r, \Omega)$ and $\bar{E}_{m}(\mathbf{r})$ is the electric field radiated by the array with an input current of $I_{0}$ into the $m$ th array element and all other elements open circuited, or the embedded open circuit loaded radiation field pattern.

In general, both $\mathbf{Z}_{A}$ and $v_{o c, m}\left(\hat{p}, E_{0}, \Omega\right)$ include antenna coupling effects. For a minimum scattering antenna, there exists a particular reactive load for which the antenna at the frequency of interest is invisible [16]. A small antenna such as a dipole below first resonance is approximately minimum scattering [17], since the fundamental mode of the antenna can be loaded in such a way that the fundamental mode does not radiate a scattered field, and higher order modes on a short dipole are excited only weakly by an incident plane wave. While an open circuit may not be exactly the reactive load required for minimum scattering, it is a close approximation. It follows that the open circuit loaded patterns for half wavelength or shorter dipoles are approximately equal to the isolated element pattern, and 
that all information about mutual coupling is contained in the impedance matrix. For non-minimum scattering antennas, $v_{o c, m}\left(\hat{p}, E_{0}, \Omega\right)$ is affected by the presence of nearby open circuit loaded elements and is different from the isolated element pattern.

If the impedance matrix looking into the loads attached to the array is $\mathbf{Z}_{R}$, then the loaded array port voltage vector is related to the open circuit voltages by the linear transformation

$$
\mathbf{v}_{R}=\underbrace{\mathbf{Z}_{R}\left(\mathbf{Z}_{R}+\mathbf{Z}_{A}\right)^{-1}}_{\mathbf{Q}} \mathbf{v}_{\mathrm{Oc}}
$$

If the receiver chains are uncoupled and identical, then the receiver output voltage vector is proportional to $\mathbf{v}_{R}$. If the receiver chains are coupled, then the receiver output voltages can be obtained from (2) by including an additional linear transformation. The receiver output voltages are combined using analog or digital beamforming to produce a scalar output signal for each beam according to

$$
v=\mathbf{w}^{H} \mathbf{v}
$$

where $\mathbf{w}$ is a vector of beamformer weights and the superscript ${ }^{H}$ denotes the Hermitian conjugate.

We assume a wide sense stationary signal and noise environment. The receiver output voltages are characterized by their correlation matrices [18-20]. If the signals $v_{m}$ are ergodic, the spatiotemporal correlation function is

$$
c_{m n}(\tau)=\lim _{T \rightarrow \infty} \frac{1}{2 T} \int_{-\infty}^{\infty} v_{m}(t) v_{n}^{*}(t-\tau) d t
$$

If the signals $v_{m}$ are spectrally white over the band of interest, or if the array signal processing does not exploit temporal correlations, then we may consider only the $\tau=0$ case, and work with the array spatial covariance matrix $R_{\mathbf{v}, m n}=c_{m n}(0)$. Evaluating the correlation function at $\tau=0$ and estimating the integral in terms of samples leads to the receiver output voltage correlation matrix

$$
\mathbf{R}_{\mathbf{v}}=\lim _{N \rightarrow \infty} \frac{1}{N} \sum_{n=1}^{N} \mathbf{v}[n] \mathbf{v}^{H}[n]
$$

The beam time average output power is

$$
P=\mathbf{w}^{H} \mathbf{R}_{\mathbf{v}} \mathbf{w}
$$

where we have ignored a factor of $1 /(2 R)$, with $R$ being the load resistance at the beamformer output. Since beamformer output powers generally appear in ratios, the scale factor is unimportant.

The array outputs consist of contributions due to the signal of interest, receiver noise, and thermal noise due to the external environment and warm antenna elements.
In terms of signal and noise correlation matrices, the signal to noise ratio (SNR) at the beamformer output is

$$
\mathrm{SNR}=\frac{\mathbf{w}^{H} \mathbf{R}_{\mathrm{sig}} \mathbf{w}}{\mathbf{w}^{H} \mathbf{R}_{\text {noise }} \mathbf{w}}
$$

If the signal of interest is a plane wave, the SNR is proportional to the beam sensitivity or $G / T$.

The noise correlation matrix $\mathbf{R}_{\text {noise }}$ consists of contributions from receiver noise, antenna element ohmic losses, and the environment around the antenna. We will consider the array to be in an isotropic noise environment with brightness temperature $T_{\text {iso }}$. This may represent thermal noise, sky noise, or interference in a richly scattering multipath environment. The correlation matrix of the array output due to the external isotropic noise is [21]

$$
\begin{aligned}
\mathbf{R}_{\text {iso }} & =\mathbf{Q E}\left[\mathbf{v}_{\text {iso }, \text { oc }} \mathbf{v}_{\text {iso }, \text { oc }}^{H}\right] \mathbf{Q}^{H} \\
& =\frac{1}{\left|I_{0}\right|^{2}} 16 k_{b} T_{\text {iso }} B \mathbf{Q} \mathbf{A} \mathbf{Q}^{H}
\end{aligned}
$$

where $\mathrm{E}[\cdot]$ represents the expectation. $\mathbf{A}$ is the pattern overlap integral matrix with elements given by

$$
A_{m n}=\frac{1}{2 \eta_{0}} \int \bar{E}_{m}(\mathbf{r}) \cdot \bar{E}_{n}^{*}(\mathbf{r}) r^{2} d \Omega
$$

where $\eta_{0}$ is the characteristic impedance of free space.

If the array is lossy, the antenna elements contribute additional internal thermal noise. If the antenna is in thermal equilibrium with the environment, the thermal noise correlation matrix is [22]

$$
\mathbf{R}_{\mathrm{t}}=8 k_{b} T_{\mathrm{iso}} B \mathbf{Q} \operatorname{Re}\left[\mathbf{Z}_{A}\right] \mathbf{Q}^{H}
$$

This can be divided into external and internal noise contributions according to

$$
\mathbf{R}_{\mathrm{t}}=\mathbf{R}_{\text {iso }}+8 k_{b} T_{\mathrm{iso}} B \mathbf{Q} \mathbf{R}_{A, \mathrm{ohmic}} \mathbf{Q}^{H}
$$

where $\mathbf{R}_{A \text {,ohmic }}$ is the ohmic part of the antenna mutual resistance. In practice, the thermal environment may not be isotropic, so that the actual thermal noise correlation matrix may be different from (11), but as will be seen below $\mathbf{R}_{t}$ still plays an important role in defining equivalent noise temperatures.

These results indicate an important connection between transmit and receive antennas. Through (8), the pattern overlap integrals determine the isotropic noise power received by the array. The pattern overlap integrals also determine the power radiated by the array when operated as a transmitter. For an arbitrary excitation current $I_{m}$ at each element port, the total power radiated by the array is

$P_{\mathrm{rad}}=\frac{1}{2 \eta_{0}\left|I_{0}\right|^{2}} \oint|\bar{E}(\mathbf{r})|^{2} r^{2} d \Omega$ 


$$
\begin{aligned}
& =\frac{1}{2 \eta_{0}\left|I_{0}\right|^{2}} \oint \sum_{m=1}^{M} I_{m} \bar{E}_{m}(\mathbf{r}) \cdot \sum_{n=1}^{M} I_{n}^{*} \bar{E}_{n}^{*}(\mathbf{r}) r^{2} d \Omega \\
& =\frac{1}{\left|I_{0}\right|^{2}} \sum_{m=1}^{M} \sum_{n=1}^{M} I_{m} I_{n}^{*} \frac{1}{2 \eta_{0}} \oint \bar{E}_{m}(\mathbf{r}) \cdot \bar{E}_{n}^{*}(\mathbf{r}) r^{2} d \Omega \\
& =\frac{1}{\left|I_{0}\right|^{2}} \mathbf{i}_{A}^{T} \mathbf{A i}_{A}^{*}
\end{aligned}
$$

where $\mathbf{i}_{A}$ is a vector of the array element port input currents. The total power accepted by the array is $P_{\text {in }}=$ $\frac{1}{2} \mathbf{i}_{A}^{H} \mathbf{R}_{A} \mathbf{i}$, where $\mathbf{R}_{A}=\operatorname{Re}\left[\mathbf{Z}_{A}\right]$, and the dissipated power is $\frac{1}{2} \mathbf{i}_{A}^{H} \mathbf{R}_{A, \text { ohmic }} \mathbf{i}$. By conservation of energy, these relationships imply that

$$
\mathbf{R}_{A, \text { ohmic }}=\mathbf{R}_{A}-\frac{2}{\left|I_{0}\right|^{2}} \mathbf{A}
$$

This can be transformed into the connection between pattern overlap integrals and the array S-parameter matrix obtained by Stein [23]. Equation (13) implies that the pattern overlap matrix $\mathbf{A}$ and the isotropic noise correlation matrix $\mathbf{R}_{\text {iso }}$ are real, which is not obvious from the definitions. For a lossless array, the pattern overlap matrix (9), the mutual resistance matrix $\mathbf{R}_{A}$, and the isotropic noise correlation matrix are all proportional. For a receiving array, (13) can be obtained using Eqs. (8), (10), and (11) based purely on noise considerations. These results are a manifestation of the fundamental connection between transmitted power and received isotropic thermal noise implied by reciprocity.

\section{AMPLIFIER NoISE Model}

We review here the classical two-port noise theory [19, 20,24], and use this to develop an amplifier noise model for the array receiver. We will first consider correlated voltage and current sources at the input port of an ideal, noiseless amplifier, as shown in Figure 1, and later in Section III-B we will transform results to the noise wave formulation.

Let the current source at one LNA be denoted by $i_{n, R}$ and the voltage source by $v_{n, R}$. The RMS densities of the current and voltage noise sources are $\bar{v}_{n, R}(\mathrm{~V} / \sqrt{\mathrm{Hz}})$ and $\bar{i}_{n, R}(\mathrm{~A} / \sqrt{\mathrm{Hz}})$. The current and voltage noise signals are partially correlated according to

$$
i_{n, R}=Y_{c} v_{n, R}+i_{u, R}
$$

where $i_{u, R}$ and $v_{n, R}$ are uncorrelated and $Y_{c}$ is the correlation admittance. We will assume the noise sources from different amplifiers are uncorrelated. For a single amplifier, the noise is minimized if the source impedance has an optimal value $Z_{\mathrm{opt}}$, which can be found in terms of the amplifier noise parameters $i_{n, R}, v_{n, R}$, and $Y_{c}$. In terms of the RMS voltage and current densities, the minimum possible equivalent temperature for a single amplifier with the optimal source impedance is

$$
T_{\min }=\frac{\bar{v}_{n, R} \bar{i}_{n, R}}{2 k_{b}}\left(\sqrt{1-c_{i}^{2}}+c_{r}\right)
$$

where the voltage and current noise correlation coefficient $c=c_{r}+j c_{i}$ is related to the correlation admittance by $Y_{c}=c \bar{i}_{n, R} / \bar{v}_{n, R}$.

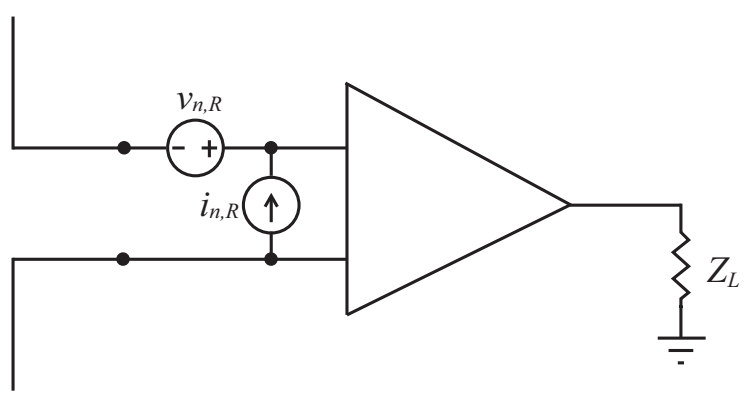

Fig. 1. Amplifier noise model for one receiver channel.

For an array, we need to determine the correlation matrix of the amplifier noise signals at the receiver outputs in terms of the amplifier noise parameters and the array and amplifier mutual impedance matrices. Let $\mathbf{v}_{n, R}$ be a vector of the noise voltages corresponding to the equivalent voltage sources for each amplifier, and $\mathbf{i}_{n, R}$ be defined similarly for the current noise signals. We will denote the vector of amplifier noise voltages across the amplifier input ports including the effects of loading by the array impedance as $\mathbf{v}_{n}$. The network relationships at the junction between the array and amplifiers are

$$
\begin{aligned}
\mathbf{v}_{n} & =\mathbf{Z}_{R} \mathbf{i}_{n} \\
\mathbf{v}_{n}-\mathbf{v}_{n, R} & =\mathbf{Z}_{A}\left(\mathbf{i}_{n, R}-\mathbf{i}_{n}\right)
\end{aligned}
$$

Solving for $\mathbf{v}_{n}$,

$$
\mathbf{v}_{n}=\underbrace{\mathbf{Z}_{R}\left(\mathbf{Z}_{R}+\mathbf{Z}_{A}\right)^{-1} \mathbf{Z}_{A}}_{\mathbf{Q} \mathbf{Z}_{A}} \mathbf{i}_{n, R}+\underbrace{\mathbf{Z}_{R}\left(\mathbf{Z}_{R}+\mathbf{Z}_{A}\right)^{-1}}_{\mathbf{Q}} \mathbf{v}_{n, R}
$$

From the noise voltage $\mathbf{v}_{n}$, we can compute the noise voltage correlation matrix. This is equivalent to the noise correlation matrix formulation of [19], except that here we use the phasor representation for signals and the convention for noise correlations is larger by a factor of two than in [19]. The amplifier noise correlation matrix is

$$
\begin{aligned}
\mathbf{R}_{\mathrm{rec}} & =\mathrm{E}\left[\mathbf{v}_{n} \mathbf{v}_{n}^{H}\right] \\
& =\mathrm{E}\left[\left(\mathbf{Q} \mathbf{Z}_{A} \mathbf{i}_{n, R}+\mathbf{Q} \mathbf{v}_{n, R}\right)\left(\mathbf{Q} \mathbf{Z}_{A} \mathbf{i}_{n, R}+\mathbf{Q} \mathbf{v}_{n, R}\right)^{H}\right] \\
& =\mathbf{Q}\left(\mathbf{Z}_{A} \mathrm{E}\left[\mathbf{i}_{n, R} \mathbf{i}_{n, R}^{H}\right] \mathbf{Z}_{A}^{H}+\mathbf{Z}_{A} \mathrm{E}\left[\mathbf{i}_{n, R} \mathbf{v}_{n, R}^{H}\right]+\right. \\
& \left.\mathrm{E}\left[\mathbf{v}_{n, R} \mathbf{i}_{n, R}^{H}\right] \mathbf{Z}_{A}^{H}+\mathrm{E}\left[\mathbf{v}_{n, R} \mathbf{v}_{n, R}^{H}\right] \mathbf{Z}_{A}^{H}\right) \mathbf{Q}^{H}
\end{aligned}
$$

Using (14) and the fact that $i_{u, R}$ and $v_{n, R}$ are uncorrelated,

$$
\begin{aligned}
\mathbf{R}_{\mathrm{rec}} & =\mathbf{Q}\left\{\mathrm{E}\left[\left|v_{n, R}\right|^{2}\right]\left(\mathbf{I}+Y_{c} \mathbf{Z}_{A}+Y_{c}^{*} \mathbf{Z}_{A}^{H}\right)+\right. \\
& \left.\mathrm{E}\left[\left|i_{n, R}\right|^{2}\right] \mathbf{Z}_{A} \mathbf{Z}_{A}^{H}\right\} \mathbf{Q}^{H}
\end{aligned}
$$


By definition of the RMS densities, $\mathrm{E}\left[\left|v_{n, R}\right|^{2}\right]=$ $2 B \bar{v}_{n, R}^{2}$ and $\mathrm{E}\left[\left|i_{n, R}\right|^{2}\right]=2 B \bar{i}_{n, R}^{2}$, where $B$ is the system noise equivalent bandwidth. We have finally

$$
\begin{aligned}
\mathbf{R}_{\mathrm{rec}} & =2 B \mathbf{Q}\left[\bar{v}_{n, R}^{2}\left(\mathbf{I}+Y_{c} \mathbf{Z}_{A}+Y_{c}^{*} \mathbf{Z}_{A}^{H}\right)+\right. \\
& \left.\bar{i}_{n, R}^{2} \mathbf{Z}_{A} \mathbf{Z}_{A}^{H}\right] \mathbf{Q}^{H}
\end{aligned}
$$

for the LNA noise correlation matrix. We will ignore in this paper the noise contribution from receiver components following the LNAs, although this could be readily included in the model if needed.

The above derivation assumed that the amplifier noise parameters are identical. We also want to consider the case where the amplifier noise parameters are different, but constrained to have the same minimum noise temperatures (15). In this case, (21) becomes

$$
\begin{aligned}
& \mathbf{R}_{\mathrm{rec}}=2 B \mathbf{Q}\left[\mathbf{V}_{n, R}^{2}+\mathbf{Z}_{A} \mathbf{Y}_{c} \mathbf{V}_{n, R}^{2}+\mathbf{V}_{n, R}^{2} \mathbf{Y}_{c}^{H} \mathbf{Z}_{A}^{H}+\right. \\
&\left.\mathbf{Z}_{A} \mathbf{I}_{n, R}^{2} \mathbf{Z}_{A}^{H}\right] \mathbf{Q}^{H}
\end{aligned}
$$

where $\mathbf{V}_{n, R}, \mathbf{I}_{n, R}$, and $\mathbf{Y}_{c}$ are diagonal matrices of noise voltage densities, noise current densities, and correlation admittances, respectively.

\section{A. Noise Matching}

One of the goals of this paper is to specify the optimal choice for the optimal source impedance $Z_{\mathrm{opt}}=$ $1 / Y_{\text {opt }}$ for each front end amplifier in a phased array system. This motivates the use of an alternative set of noise parameters, $T_{\min }, R_{n}$, and $Y_{\text {opt }}$, where the noise resistance is defined by

$$
R_{n}=\frac{\bar{v}_{n, R}^{2}}{4 k_{b} T_{0}}
$$

Since the noise resistance specifies the sensitivity of the amplifier noise figure to changes in the source impedance, and the effective impedance seen by each amplifier source depends on the beam steering direction, we will see that for a phased array, $R_{n}$ is a critical amplifier design parameter. In practice, amplifier noise parameters are linked by a particular design topology and are not independent, but for the purposes of this study, it is important to maintain constant values for $R_{n}$ and $T_{\min }$ while varying $Z_{\mathrm{opt}}$, in order to separate noise matching effects from amplifier performance effects.

To facilitate the noise matching procedure, it is convenient to express the noise parameters $\bar{v}_{n, R}, \bar{i}_{n, R}$, and $Y_{c}$ in terms of $T_{\min }, R_{n}$, and $Y_{\text {opt }}$. From the two-port noise model, it can be shown that

$$
\begin{aligned}
Y_{c} & =\frac{T_{\min }}{2 R_{n} T_{0}}-Y_{\mathrm{opt}} \\
\bar{i}_{n, R}^{2} & =\left|Y_{\mathrm{opt}}\right|^{2} \bar{v}_{n, R}^{2}
\end{aligned}
$$

These relationships provide a transformation from one set of noise parameters to the other. Although the transistor noise model has four real, independent parameters, (14) imposes a constraint on the range of possible values of the parameters. Physically, the correlated part of the current noise cannot be greater than the total current noise, from which it follows with (25) that $\left|Y_{c}\right| \leq\left|Y_{\text {opt }}\right|$. In view of (24), the noise resistance is therefore bounded below by

$$
R_{n} \geq \frac{T_{\mathrm{min}}}{4 G_{\mathrm{opt}} T_{0}}
$$

where $Y_{\mathrm{opt}}=G_{\mathrm{opt}}+j B_{\mathrm{opt}}$.

\section{B. Noise Wave Formulation}

In the above treatment, we have formulated the amplifier noise model in terms of mutual impedances. The voltage and current noise source model has a close relationship to the physical processes in a transistor that produce amplifier noise, but for an S-parameter based network analysis, we must reformulate the results in terms of noise waves [20,24].

In converting from the current and voltage noise sources of Figure 1 to the noise wave model, we make use of the equivalent noise resistance (23) and the uncorrelated current noise conductance

$$
G_{u}=\frac{\bar{i}_{u, R}^{2}}{4 k_{b} T_{0}}
$$

where $i_{u}$ is defined by (14). The noise wave parameters are [24]

$$
\begin{aligned}
& T_{\alpha}=T_{0}\left[Z_{0} G_{u}+R_{n} Y_{0}\left(1+Z_{0}^{2}\left|Y_{c}\right|^{2}+2 Z_{0} G_{c}\right)\right] \\
& T_{\beta}=T_{0}\left[Z_{0} G_{u}+R_{n} Y_{0}\left(1+Z_{0}^{2}\left|Y_{c}\right|^{2}-2 Z_{0} G_{c}\right)\right] \\
& T_{\gamma}=T_{0}\left[-Z_{0} G_{u}+R_{n} Y_{0}\left(1-Z_{0}^{2}\left|Y_{c}\right|^{2}\right)-j 2 R_{n} B_{c}\right]
\end{aligned}
$$

where the first two are real and the third is complex. In some treatments of amplifier noise wave parameters, $T_{\gamma}$ is separated into a magnitude and phase, but here we include the phase in the definition of $T_{\gamma}$. In terms of the noise wave parameters,

$$
\begin{aligned}
& T_{\min }=\frac{1}{2}\left(T_{\alpha}-T_{\beta}+\sqrt{\left(T_{\alpha}+T_{\beta}\right)^{2}-4\left|T_{\gamma}\right|^{2}}\right) \\
& \Gamma_{\mathrm{opt}}=\frac{1}{2 T_{\gamma}}\left(T_{\alpha}+T_{\beta}-\sqrt{\left(T_{\alpha}+T_{\beta}\right)^{2}-4\left|T_{\gamma}\right|^{2}}\right)
\end{aligned}
$$

The noise wave parameters are related to the forward and reverse noise waves produced by the amplifier 
according to

$$
\begin{aligned}
& k_{b} T_{\alpha} B=\frac{1}{2} \mathrm{E}\left[\left|a_{\eta}\right|^{2}\right] \\
& k_{b} T_{\beta} B=\frac{1}{2} \mathrm{E}\left[\left|b_{\eta}\right|^{2}\right] \\
& k_{b} T_{\gamma} B=\frac{1}{2} \mathrm{E}\left[a_{\eta}^{*} b_{\eta}\right]
\end{aligned}
$$

where $a_{\eta}$ and $b_{\eta}$ are the forward and reverse noise wave amplitudes. The network relationships including vectors of noise waves from each amplifier are

$$
\begin{aligned}
\mathbf{a} & =\mathbf{S}_{A} \mathbf{b}-\mathbf{a}_{\eta} \\
\mathbf{b} & =\mathbf{S}_{R} \mathbf{a}+\mathbf{b}_{\eta}
\end{aligned}
$$

where the negative sign in the first equation is by convention. By eliminating $\mathbf{b}$, the total forward noise wave is found to be

$$
\mathbf{a}=\left(\mathbf{I}-\mathbf{S}_{A} \mathbf{S}_{R}\right)^{-1}\left(\mathbf{S}_{A} \mathbf{b}_{\eta}-\mathbf{a}_{\eta}\right)
$$

The voltage at the amplifier inputs is

$$
\mathbf{v}=\underbrace{Z_{0}^{1 / 2}\left(\mathbf{I}+\mathbf{S}_{R}\right)\left(\mathbf{I}-\mathbf{S}_{A} \mathbf{S}_{R}\right)^{-1}}_{\mathbf{G}}\left(\mathbf{S}_{A} \mathbf{b}_{\eta}-\mathbf{a}_{\eta}\right)
$$

The matrix $\mathbf{G}$ is similar to $\mathbf{Q}$ in (2) but transforms forward waves into the amplifier inputs in the case of matched ports to receiver output voltages. Using (31), the amplifier noise correlation matrix is

$$
\mathbf{R}_{\mathrm{rec}}=2 k_{b} B \mathbf{G} \mathbf{R}_{\eta} \mathbf{G}^{H}
$$

where

$$
\mathbf{R}_{\eta}=\mathbf{T}_{\alpha}+\mathbf{S}_{A} \mathbf{T}_{\beta} \mathbf{S}_{A}^{H}-\mathbf{S}_{A} \mathbf{T}_{\gamma}-\mathbf{T}_{\gamma}^{H} \mathbf{S}_{A}^{H}
$$

and $\mathbf{T}_{\alpha}, \mathbf{T}_{\beta}$, and $\mathbf{T}_{\gamma}$ are diagonal matrices of the noise wave parameters for each amplifier.

\section{Beam Equivalent Receiver Noise TEMPERATURE AND NoISE MATCHING EFFICIENCY}

For a passive receiving antenna, the conventions for effective area and antenna noise temperature are based on available powers at the antenna terminals [25]. For a beamforming array with unequal gains and combining coefficients in the signal paths, an equivalent passive antenna can be defined using the noise response of the array in a thermal environment at a given temperature $T_{\text {iso }}[7$, $8,21]$. Using this approach, the equivalent receiver noise temperature referred to available power at the terminals of a beam-equivalent passive feed is

$$
T_{\text {rec }}=T_{\text {iso }} \frac{\mathbf{w}^{H} \mathbf{R}_{\text {rec }} \mathbf{w}}{\mathbf{w}^{H} \mathbf{R}_{\mathrm{t}} \mathbf{w}}
$$

where $\mathbf{R}_{t}$ is defined in (10). This expression refers the receiver noise to an equivalent antenna noise temperature, so that the beam equivalent receiver noise temperature is the physical temperature of the environment and array elements which would be required to make a noise power contribution at the beamformer output equal to that of the noisy receivers.

Using the minimum receiver noise temperature under optimal matching conditions, a noise matching efficiency can be defined by

$$
\eta_{\mathrm{n}}=\frac{T_{\min }}{T_{\mathrm{rec}}}
$$

In terms of noise correlation matrices, the noise matching efficiency is

$$
\eta_{\mathrm{n}}=\frac{T_{\min }}{T_{\text {iso }}} \frac{\mathbf{w}^{H} \mathbf{R}_{\mathrm{t}} \mathbf{w}}{\mathbf{w}^{H} \mathbf{R}_{\mathrm{rec}} \mathbf{w}}
$$

This efficiency factor measures the noise penalty caused by amplifier mismatch and array mutual coupling. For an uncoupled array with each front end amplifier optimally matched to an antenna element, $\eta_{\mathrm{n}}=1$. If the array is coupled or the amplifiers are not optimally matched to the individual antenna elements, then in general $\eta_{\mathrm{n}}<1$.

Using Eqs. (10) and (22) in (38), the beam equivalent receiver noise temperature can be given in terms of the amplifier noise parameters and array mutual resistance matrix as

$$
T_{\text {rec }}=\frac{1}{4 k_{b}} \frac{\mathbf{w}^{H} \mathbf{Q} \mathbf{R}_{\mathrm{rec}, \mathrm{oc}} \mathbf{Q}^{H} \mathbf{w}}{\mathbf{Q} \operatorname{Re}\left[\mathbf{Z}_{A}\right] \mathbf{Q}^{H} \mathbf{w}}
$$

where

$$
\mathbf{R}_{\mathrm{rec}, \mathrm{oc}}=\mathbf{V}_{n, R}^{2}+\mathbf{Z}_{A} \mathbf{Y}_{c} \mathbf{V}_{n, R}^{2}+\mathbf{V}_{n, R}^{2} \mathbf{Y}_{c}^{H} \mathbf{Z}_{A}^{H}+\mathbf{Z}_{A} \mathbf{I}_{n, R}^{2} \mathbf{Z}_{A}^{H}
$$

To simplify this expression, we can define

$$
\mathbf{w}_{\mathrm{oc}}=\mathbf{Q}^{H} \mathbf{w}
$$

which can be interpreted as the beamformer weights which give the beam output when applied to open circuit voltages at the array element terminals. The beam receiver noise temperature becomes

$$
T_{\mathrm{rec}}=\frac{1}{4 k_{b}} \frac{\mathbf{w}_{\mathrm{oc}}^{H} \mathbf{R}_{\mathrm{rec}, \mathrm{oc}} \mathbf{w}_{\mathrm{oc}}}{\mathbf{w}_{\mathrm{oc}}^{H} \operatorname{Re}\left[\mathbf{Z}_{A}\right] \mathbf{w}_{\mathrm{oc}}}
$$

Using this receiver noise model, the noise matching efficiency is

$$
\eta_{\mathrm{n}}=4 k_{b} T_{\min } \frac{\mathbf{w}_{\mathrm{oc}}^{H} \operatorname{Re}\left[\mathbf{Z}_{A}\right] \mathbf{w}_{\mathrm{oc}}}{\mathbf{w}_{\mathrm{oc}}^{H} \mathbf{R}_{\mathrm{rec}, \mathrm{oc}} \mathbf{w}_{\mathrm{oc}}}
$$

Using the noise wave formulation, a simple expression for the beam equivalent amplifier noise temperature can be developed. The correlation of forward noise waves produced by a lossy network in thermal equilibrium with an environment at temperature $T_{\text {iso }}$ is given by Bosma's theorem [26, 27]

$$
\mathbf{R}_{\mathrm{t}, \mathrm{f}}=2 k_{b} T_{\mathrm{iso}} B\left(\mathbf{I}-\mathbf{S}_{A} \mathbf{S}_{A}^{H}\right)
$$


Using the transformation defined in (35), we can use Bosma's theorem to express (11) in terms of Sparameters:

$$
\mathbf{R}_{\mathrm{t}}=2 k_{b} T_{\mathrm{iso}} B \mathbf{G}\left(\mathbf{I}-\mathbf{S}_{A} \mathbf{S}_{A}^{H}\right) \mathbf{G}^{H}
$$

From (38) and (36) the beam equivalent receiver noise temperature is

$$
T_{\text {rec }}=\frac{\mathbf{w}^{H} \mathbf{G} \mathbf{R}_{\eta} \mathbf{G}^{H} \mathbf{w}}{\mathbf{w}^{H} \mathbf{G}\left(\mathbf{I}-\mathbf{S}_{A} \mathbf{S}_{A}^{H}\right) \mathbf{G}^{H} \mathbf{w}}
$$

Since all port quantities, whether currents, voltages, or wave amplitudes under any given loading condition are related by linear transformations, we can specify the beamformer equivalently in terms of any of these quantities. Equation (48) can be simplified by defining $\mathbf{w}_{\mathrm{f}}=\mathbf{G}^{H} \mathbf{w}$, which is the beamformer weight vector relative to the forward wave amplitudes at the amplifier input ports if the ports were matched. Using the forward wave beamformer weight vector, the beam equivalent receiver noise temperature is

$$
T_{\text {rec }}=\frac{\mathbf{w}_{\mathrm{f}}^{H}\left(\mathbf{T}_{\alpha}+\mathbf{S}_{A} \mathbf{T}_{\beta} \mathbf{S}_{A}^{H}-\mathbf{S}_{A} \mathbf{T}_{\gamma}-\mathbf{T}_{\gamma}^{H} \mathbf{S}_{A}^{H}\right) \mathbf{w}_{\mathrm{f}}}{\mathbf{w}_{\mathrm{f}}^{H}\left(\mathbf{I}-\mathbf{S}_{A} \mathbf{S}_{A}^{H}\right) \mathbf{w}_{\mathrm{f}}}
$$

This result generalizes the single amplifier noise temperature to a phased array antenna, in terms of the array S-parameter matrix and the amplifier noise wave parameters.

\section{A. Approximate Noise Model}

To provide insight into the behavior of the noise matching efficiency, we will consider the special case for which the array is lossless, the mutual impedance matrix is real $\left(\mathbf{Z}_{A}=\mathbf{R}_{A}\right)$, the current and voltage noise sources are uncorrelated $\left(Y_{c}=0\right)$, and the amplifiers are noise matched to the array self impedances. The latter condition implies that $\mathbf{V}_{n, R}=\mathbf{R}_{A}^{d} \mathbf{I}_{n, R}$, where $\mathbf{R}_{A}^{d}=\operatorname{diag}\left(\mathbf{R}_{A}\right)$.

Under these assumptions, (22) becomes

$$
\mathbf{R}_{\mathrm{rec}}=2 B \mathbf{Q}\left[\mathbf{V}_{n, R}^{2}+\mathbf{R}_{A} \mathbf{I}_{n, R}^{2} \mathbf{R}_{A}\right] \mathbf{Q}^{H}
$$

By (15) with $Y_{c}=0$, we have $\mathbf{R}_{A}^{d} \mathbf{I}_{n, R}^{2}=2 k_{B} T_{\min } \mathbf{I}$. Inserting this in (50) leads to

$$
\mathbf{R}_{\mathrm{rec}}=2 B \mathbf{Q}\left[\mathbf{R}_{A}^{d}+\mathbf{R}_{A} \mathbf{R}_{A}^{d-1} \mathbf{R}_{A}\right] \mathbf{Q}^{H}
$$

For a lossless array, $\mathbf{R}_{\mathrm{t}}=\frac{1}{\left|I_{0}\right|^{2}} 16 k_{b} T_{\text {iso }} B \mathbf{A}$ and $\mathbf{R}_{A}=\frac{2}{\left|I_{0}\right|^{2}} \mathbf{A}$. The noise matching efficiency then becomes

$$
\eta_{\mathrm{n}}=\frac{\mathbf{w}_{\mathrm{oc}}^{H} \mathbf{A} \mathbf{w}_{\mathrm{oc}}}{\mathbf{w}_{\mathrm{oc}}^{H} \frac{1}{2}\left(\mathbf{A}_{d}+\mathbf{A} \mathbf{A}_{d}{ }^{-1} \mathbf{A}\right) \mathbf{w}_{\mathrm{oc}}}
$$

where $\mathbf{A}_{d}=\operatorname{diag}(\mathbf{A})$. This expression is based on restrictive assumptions about the amplifier noise parameters, but has an advantage over the general definition
(40) in that it is intrinsic to the antenna and beamformer weights. The noise matching efficiency (52) is optimistic, because $Y_{c}=0$ together with (24) implies that $R_{n}$ is only twice the minimum value in (26). For $T_{\min }=20 \mathrm{~K}$ and element self-impedances of $50 \Omega$, this leads to $R_{n}=$ $1.7 \Omega$, which is a relatively small value at microwave frequencies.

If the array mutual resistances are identical (which implies that the diagonal elements of $\mathbf{A}$ are identical), then the open circuit receiver noise correlation matrix simplifies to

$$
\mathbf{R}_{\mathrm{rec}}=2 k_{b} B T_{\min } R_{A, 11} \mathbf{Q} \frac{1}{2}\left(\mathbf{I}+\tilde{\mathbf{A}}^{2}\right) \mathbf{Q}^{H}
$$

where $\tilde{\mathbf{A}}$ is the overlap integral matrix normalized such that the diagonal elements are unity, so that the elements of $\tilde{\mathbf{A}}$ are given by

$$
\tilde{A}_{m n}=\frac{R_{A, m, n}}{R_{A, m, m}}
$$

The noise matching efficiency becomes

$$
\eta_{\mathrm{n}}=\frac{\mathbf{w}_{\mathrm{oc}}^{H} \tilde{\mathbf{A}} \mathbf{w}_{\mathrm{oc}}}{\mathbf{w}_{\mathrm{oc}}^{H} \frac{1}{2}\left(\mathbf{I}+\tilde{\mathbf{A}}^{2}\right) \mathbf{w}_{\mathrm{oc}}}
$$

By making the transformation $\mathbf{w}^{\prime}=\tilde{\mathbf{A}}^{1 / 2} \mathbf{w}_{\mathrm{oc}}$, this can be placed in the form

$$
\eta_{\mathrm{n}}=\frac{\mathbf{w}^{\prime H} \mathbf{w}^{\prime}}{\mathbf{w}^{\prime H} \frac{1}{2}\left(\tilde{\mathbf{A}}^{-1}+\tilde{\mathbf{A}}\right) \mathbf{w}^{\prime}}
$$

Since $\tilde{\mathbf{A}}$ is a normal matrix, the range of possible values of the quadratic form (56) is the convex hull of the inverse eigenvalues of $\frac{1}{2}\left(\tilde{\mathbf{A}}^{-1}+\tilde{\mathbf{A}}\right)$. Since $\tilde{\mathbf{A}}$ is real and symmetric, the eigenvalues are real. Furthermore, on physical grounds it is not possible for any beamformer to realize infinite SNR with an isotropic noise field, so from (8) and (54) the matrix $\tilde{\mathbf{A}}$ must be positive definite. This implies that the eigenvalues of $\frac{1}{2}\left(\tilde{\mathbf{A}}+\tilde{\mathbf{A}}^{-1}\right)$ are greater than or equal to one, and the noise matching efficiency is therefore between zero and one. If the array is uncoupled, then $\tilde{\mathbf{A}}=\mathbf{I}$, and the noise matching efficiency is unity.

\section{B. Close Element Spacing}

For small, closely spaced antenna elements, the approximation (52) for the noise matching efficiency has a closed form limit. For isotropic radiators, the pattern overlap integrals are

$$
A_{11}=A_{22}=\frac{1}{2 \eta} \oint\left|\bar{E}_{1}\right|^{2} r^{2} d \Omega=P_{\mathrm{rad}}
$$


and

$$
\begin{aligned}
A_{12}=A_{21} & =\frac{P_{\mathrm{rad}}}{4 \pi} \oint e^{-j \mathbf{k} \cdot \mathbf{r}} d \Omega \\
& =P_{\mathrm{rad}} \frac{\sin (k d)}{k d}
\end{aligned}
$$

where $d$ is the element separation.

For an array with closely spaced elements, by expanding (57) the overlap matrix has the form

$$
\tilde{\mathbf{A}}=\left[\begin{array}{cc}
1 & 1-\gamma \\
1-\gamma & 1
\end{array}\right]
$$

where $\gamma$ is small and vanishes as $d \rightarrow 0$. In the limit as the element spacing becomes zero, the noise matching efficiency becomes

$$
\eta_{\mathrm{n}}=\frac{\mathbf{w}_{\mathrm{oc}}^{H}\left[\begin{array}{ll}
1 & 1 \\
1 & 1
\end{array}\right] \mathbf{w}_{\mathrm{oc}}}{\mathbf{w}_{\mathrm{oc}}^{H}\left[\begin{array}{cc}
3 / 2 & 1 \\
1 & 3 / 2
\end{array}\right] \mathbf{w}_{\mathrm{oc}}}
$$

There are two cases to consider, the even mode beamformer for which $\mathbf{w}_{\mathrm{oc}}=\left[\begin{array}{ll}1 & 1\end{array}\right]^{T}$, and the odd mode beamformer with $\mathbf{w}_{\mathrm{oc}}=\left[\begin{array}{ll}1 & -1\end{array}\right]^{T}$. In the former case, $\eta_{\mathrm{n}}=0.8$, and in the latter case $\eta_{\mathrm{n}} \rightarrow 0$. This result neglects the mutual reactance of the array, which will have a considerable effect on the noise performance of the amplifiers for close element spacings, but it does give a useful qualitative picture of array noise performance as the element spacing becomes small.

\section{Optimal Noise MATCHING FOR ARRAYS}

Several array noise matching conditions have been studied previously, including the multiport decoupling network of [6], the self impedance matching condition, and the active impedance matching condition of [1113]. After reviewing the latter two of these approaches, we will present a new matching condition for phased arrays that optimizes the system noise performance over multiple beam steering directions. We will also develop a matrix formulation which allows noise performance to be characterized for a non-beamformed array.

\section{A. Active Impedance Matching Condition}

For a given beamformer, it has been shown that the receiver noise can be minimized and a noise matching efficiency of unity achieved by noise matching the amplifiers to the active impedance or active reflection coefficient at each element port [11-13]. Consider the amplifier noise voltage signal

$$
v=\mathbf{w}_{\mathrm{f}}^{H}\left(\mathbf{S}_{A} \mathbf{b}_{\eta}-\mathbf{a}_{\eta}\right)
$$

at the beamformer output. The noise contribution from the $m$ th amplifier is

$$
v_{m}=w_{\mathrm{f}, m}^{*}\left(\Gamma_{\mathrm{a}, m} b_{\eta, m}-a_{\eta, m}\right)
$$

where the active reflection coefficient is defined to be

$$
\Gamma_{\mathrm{a}, m}=\frac{1}{w_{\mathrm{f}, m}^{*}} \sum_{n=1}^{M} w_{\mathrm{f}, n}^{*} S_{A, n m}
$$

We can also define in a similar way the active impedance

$$
Z_{\mathrm{a}, m}=\frac{1}{w_{\mathrm{f}, m}^{*}} \sum_{n=1}^{M} w_{\mathrm{f}, n}^{*} Z_{A, n m}
$$

Effectively, the noise appears at the beamformer output as if the reverse wave $b_{\eta, m}$ had reflected from the active reflection coefficient $\Gamma_{\mathrm{a}, m}$. Therefore, we can noise match the amplifier to the active reflection coefficient and the noise contribution from that amplifier will be minimized. If this is done for each amplifier, the overall receiver noise temperature will be $T_{\min }$ (assuming identical amplifiers) and the noise matching efficiency will be unity.

To facilitate the analysis of the next section, we will express the beam equivalent noise temperature in terms of the active reflection coefficients or active impedances. By making use of (62) in (49), it can be shown that

$$
\begin{aligned}
T_{\mathrm{rec}} & =\frac{\sum_{m=1}^{M}\left|w_{f, m}\right|^{2} T_{\eta, m}}{\sum_{m=1}^{M}\left|w_{f, m}\right|^{2}\left(1-\left|\Gamma_{\mathrm{a}, m}\right|^{2}\right)} \\
T_{\eta, m} & =\left(\left|\Gamma_{\mathrm{a}, m}\right|^{2} T_{\beta, m}-\Gamma_{\mathrm{a}, m} T_{\gamma, m}-\Gamma_{\mathrm{a}, m}^{*} T_{\gamma, m}^{*}+T_{\alpha, m}\right)
\end{aligned}
$$

By completing the square in the numerator of (38), it can be shown that

$$
T_{\text {rec }}=T_{\text {min }}+\frac{\sum_{m=1}^{M}\left|w_{f, m}\right|^{2} T_{r, m}\left|\Gamma_{\mathrm{a}, m}-\Gamma_{\mathrm{opt}, m}\right|^{2}}{\sum_{m=1}^{M}\left|w_{f, m}\right|^{2}\left(1-\left|\Gamma_{\mathrm{a}, m}\right|^{2}\right)}
$$

where $T_{r, m}$ is the noise temperature parameter for the $m$ th amplifier defined in [12] as

$T_{r}=T_{\alpha}+T_{\beta}+\sqrt{\left(T_{\alpha}+T_{\beta}\right)^{2}-4\left|T_{\gamma}\right|^{2}}=\frac{4 R_{n} T_{0}}{Z_{0}\left|1+\Gamma_{\mathrm{opt}}\right|^{2}}$

Equation (65) can be formulated equivalently in terms of active impedances and admittances as

$$
\begin{gathered}
T_{\mathrm{rec}}=T_{\mathrm{min}}+T_{0} \frac{\sum_{m=1}^{M}\left|w_{\mathrm{oc}, m}\right|^{2} R_{c, m}}{\sum_{m=1}^{M}\left|w_{\mathrm{oc}, m}\right|^{2} R_{\mathrm{a}, m}} \\
R_{c, m}=R_{n, m}\left|Z_{\mathrm{a}, m}\right|^{2}\left|Y_{\mathrm{a}, m}-Y_{\mathrm{opt}, m}\right|^{2}
\end{gathered}
$$

where $R_{n, m}$ is the noise resistance parameter given by (23) for the $m$ th amplifier and $R_{\mathrm{a}, m}=\operatorname{Re}\left[Z_{\mathrm{a}, m}\right]$. Here, we acknowledge again the important contribution of Woestenberg [12] to the noise theory of phased array antennas. Equation (65) is very similar to a result obtained previously in that work for one amplifier in an array system, with the only major differences being that (65) combines the individual amplifier contributions into an overall beam equivalent receiver noise temperature, and a restriction that amplifier input reflection coefficients are small is removed. 


\section{B. Self Impedance Matching Condition}

If mutual coupling is small, then the off-diagonal elements of $\mathbf{Z}_{A}$ are small relative to the diagonal elements, and the active impedances (63) reduce to the diagonal elements of the impedance matrix. In this case, optimal noise performance occurs when the front end amplifiers are noise matched to the self impedances according to

$$
Z_{\mathrm{opt}, m}=Z_{A, m m}
$$

This noise matching condition requires that the optimal source impedance for the $m$ th front end amplifier be equal to the $m$ th array element self impedance $Z_{A, m m}$, or equivalently that the self impedance is transformed using a matching network to the optimal source impedance for the amplifier.

\section{Multi-Beam Average Noise Optimization}

From the preceding considerations, it is clear that the effective receiver noise temperature for a coupled array depends on the beamformer weights and the beam steering direction. For a multi-beam system, it is desirable to minimize the receiver noise over all beams. One way to do this is to decouple the array [6]. As observed in Section I, the added loss and bandwidth reduction of the decoupling network may preclude this approach. If front end amplifiers must be noise matched to a coupled array, it is desirable to have an approach for optimally noise matching the amplifiers so that the receiver noise is minimized in an average sense over the beams.

We will consider the noise resistance $R_{n}$ and minimum equivalent noise temperature $T_{\min }$ to be fixed and identical for all amplifiers, and vary $Y_{\text {opt }}$ for each amplifier to achieve the smallest average beam equivalent receiver noise temperature. This can be considered a "diagonal matching condition" in the sense of [6].

To find the beam average optimal noise matching condition, we define the average beam equivalent noise temperature to be

$$
T_{\mathrm{av}}=\frac{1}{P} \sum_{p=1}^{P} T_{r e c}^{p}
$$

where $p$ indexes the $P$ desired beams. Inserting (67) leads to

$$
T_{\mathrm{av}}=T_{\min }+\frac{T_{0}}{P} \sum_{p=1}^{P} \frac{\sum_{m=1}^{M}\left|w_{\mathrm{oc}, m}^{p}\right|^{2} R_{c, m}^{p}}{\sum_{m=1}^{M}\left|w_{\mathrm{oc}, m}^{p}\right|^{2} R_{\mathrm{a}, m}^{p}}
$$

where $R_{c, m}^{p}$ is given by (68), $\mathbf{w}_{\mathrm{oc}, m}^{p}$ are the components of the $p$ th beamformer weight vector $\mathbf{w}_{\mathrm{Oc}}^{p}$, and the weights are taken with respect to open circuit signal voltages at the antenna ports according to (43). To minimize this expression, we differentiate with respect to $Y_{\mathrm{opt}, m}^{*}$ to obtain

$$
\frac{\partial T_{\mathrm{av}}}{\partial Y_{\mathrm{opt}, m}^{*}}=\frac{T_{0}}{P} \sum_{p=1}^{P} \frac{\left|w_{\mathrm{oc}, m}^{p}\right|^{2} R_{n, m}^{p}\left|Z_{\mathrm{a}, m}^{p}\right|^{2}\left(Y_{\mathrm{opt}, m}-Y_{\mathrm{a}, m}^{p}\right)}{\left|w_{\mathrm{oc}, m}^{p}\right|^{2} R_{\mathrm{a}, m}^{p}}
$$

Setting the partial derivative to zero and solving for the optimal noise admittance leads to

$$
Y_{\mathrm{opt}, m}=\frac{\sum_{p=1}^{P}\left|w_{\mathrm{oc}, m}^{p}\right|^{2} Z_{\mathrm{a}, m}^{p *}}{\sum_{p=1}^{P}\left|w_{\mathrm{oc}, m}^{p}\right|^{2}\left|Z_{\mathrm{a}, m}^{p}\right|^{2}}
$$

This result provides an amplifier noise matching condition which minimizes the average beam equivalent receiver noise temperature given $P$ beamformer weight vectors $\mathbf{w}^{p}$.

\section{Available Amplifier Noise Correlation Matrix}

For most array applications, multiple beams are formed, either by electronic control of analog phase shifters for beam scanning, or by using digital signal processing to form multiple simultaneous beams. For other applications such as MIMO communications, beams are not formed in the usual sense, but receiver noise is still a function of how array outputs are combined in signal processing. To address noise performance for nonbeamforming arrays, we will derive a noise matrix with a spectrum that determines SNR performance for an array in a beam-independent way.

If we make a further transformation of the beamformer weight vector in (49) to $\mathbf{w}_{\mathrm{a}}=\left(\mathbf{I}-\mathbf{S}_{A} \mathbf{S}_{A}^{H}\right)^{1 / 2} \mathbf{w}_{\mathrm{f}}$, then the beam equivalent receiver noise temperature becomes

$$
T_{\text {rec }}=\frac{\mathbf{w}_{\mathrm{a}}^{H} \mathbf{R}_{\mathrm{rec}, \mathrm{a}} \mathbf{w}_{\mathrm{a}}}{\mathbf{w}_{\mathrm{a}}^{H} \mathbf{w}_{\mathrm{a}}}
$$

where

$$
\mathbf{R}_{\mathrm{rec}, \mathrm{a}}=\mathbf{M}^{H}\left(\mathbf{T}_{\alpha}+\mathbf{S}_{A} \mathbf{T}_{\beta} \mathbf{S}_{A}^{H}-\mathbf{S}_{A} \mathbf{T}_{\gamma}-\mathbf{S}_{A}^{H} \mathbf{T}_{\gamma}^{H}\right) \mathbf{M}
$$

and $\mathbf{M}=\left(\mathbf{I}-\mathbf{S}_{A} \mathbf{S}_{A}^{H}\right)^{-1 / 2}$. The elements of this matrix have units of temperature (Kelvin). $\mathbf{R}_{\text {rec,a }}$ can be considered as an available receiver noise temperature correlation matrix, and is identical to the exchangeable amplifier noise correlation matrix defined in [6] (exchangeable power is a generalization of available power which applies to active sources).

Equation (74) has a number of interesting implications for the characterization of amplifier noise matching conditions. Most importantly, the performance of a given amplifier noise matching conditions can be understood in terms of the eigenvalues of $\mathbf{R}_{\text {rec,a }}$. The range of possible values of the quadratic form (74) is the field of values of the matrix $\mathbf{R}_{\text {rec,a }}$. For a normal matrix, the field of values is the convex hull of the eigenvalues in 
the complex plane. Correlation matrices are Hermitian, real and positive semidefinite, from which it follows that the eigenvalues are real and nonnegative. Since it is not possible to achieve a beam equivalent receiver noise temperature of zero, $\mathbf{R}_{\text {rec,a }}$ is positive definite, and all the eigenvalues are positive. The field of values of $\mathbf{R}_{\text {rec,a }}$ is therefore an interval on the positive real axis from the smallest to the largest eigenvalue. The eigenvalues $T_{1} \leq T_{2} \leq \cdots \leq T_{M}$ have units of temperature, and for a given beamformer, the beam equivalent noise temperature lies between the smallest and largest eigenvalues of $\mathbf{R}_{\text {rec,a }}$, so that $T_{1} \leq T_{\text {rec }} \leq T_{M}$.

For a multiport matching network which decouples the array and optimally noise matches each decoupled port to an amplifier, the eigenvalues of $\mathbf{R}_{\text {rec,a }}$ are simultaneously minimized [6]. From this, it follows that the eigenvalues of are bounded below by $T_{\min }$ in general, and are all equal to $T_{\min }$ for the optimal multiport matching network. In consequence, both the matrix trace and determinant are minimized by the optimal multiport matching network:

$$
\begin{aligned}
\operatorname{det} \mathbf{R}_{\mathrm{rec}, \mathrm{a}} & \geq \operatorname{det} \mathbf{R}_{\mathrm{rec}, \mathrm{a}, \mathrm{opt}}=T_{\mathrm{min}}^{M} \\
\operatorname{trace} \mathbf{R}_{\mathrm{rec}, \mathrm{a}} & \geq \operatorname{trace} \mathbf{R}_{\mathrm{rec}, \mathrm{a}, \mathrm{opt}}=M T_{\mathrm{min}}
\end{aligned}
$$

where $M$ is the number of array elements and $\mathbf{R}_{\text {rec,a,opt }}$ is the available amplifier noise correlation matrix obtained with the optimal multiport matching network as defined in [6]. In general, a good amplifier noise matching condition will cause the eigenvalues of $\mathbf{R}_{\text {rec,a }}$ to be as close to $T_{\min }$ as possible. For a coupled array with any other matching condition, at least one of the eigenvalues must be larger than $T_{\min }$, and therefore there exists at least one beamformer with a beam receiver noise temperature greater than $T_{\min }$ and a receiver noise matching efficiency $\eta_{\mathrm{n}}<1$.

The condition number of a Hermitian, positive definite matrix is the ratio of the largest eigenvalue to the smallest eigenvalue. For the available receiver noise temperature correlation matrix, the condition number is

$$
\text { cond } \mathbf{R}_{\mathrm{rec}, \mathrm{a}}=\frac{T_{\mathrm{M}}}{T_{1}}
$$

The condition number of $R_{\text {rev,av,opt }}$ is unity, since all the eigenvalues are equal to $T_{\min }$. If the condition number of $\mathbf{R}_{\text {rec,a }}$ is large, then over different beamformers the beam equivalent receiver noise temperature can vary significantly. If the condition number is close to unity, then the sensitivity of the system to beam steering direction is small, and $T_{\text {rec }}$ does not vary much from one beam to another.

For a radiometric detection application such as phased array feeds for radio astronomical observations, high sensitivity requires a stable system noise response. Since the signal of interest is typically many tens of $\mathrm{dB}$ below the noise floor, the signal is detected as a small perturbation of a time-averaged noise power estimate. Unstable noise response leads to increased variance in the noise power estimate and reduces sensitivity. For an adaptive beamforming system, achieving a stable noise response as the beamformer changes over time requires that the condition number of $\mathbf{R}_{\text {rec,a }}$ be small. If the beamformer weight vectors for a multi-beam system are such that $\mathrm{w}_{\mathrm{a}}$ ranges over a subspace smaller than the full $M$-dimensional complex vector space in which $\mathbf{w}_{\mathrm{a}}$ lies, then only the eigenvalue spread over the subspace spanned by the beamformer weight vectors is significant in determining the sensitivity of equivalent receiver noise temperature to the beam steering direction.

The active impedance or active reflection coefficient matching condition reduces one of the eigenvalues of $\mathbf{R}_{\text {rec,a }}$ to $T_{\min }$, but other eigenvalues are larger than $T_{\min }$. As a result, the noise matching efficiency is less than unity when the beam is steered away from the particular beamformer used in (62) to design the amplifier noise matching conditions. In general, neither the self impedance match nor the average optimal match specified in (73) reduces any of the eigenvalues of $\mathbf{R}_{\text {rec,a }}$ to $T_{\min }$, but the eigenvalue spread may be smaller than is the case for the active impedance match. One of the main questions to be answered in numerical studies is how the eigenvalue spread of $\mathbf{R}_{\text {rec,a }}$ compares for the different matching conditions, since this determines the overall noise performance for multiple beams.

For a closely spaced array, $\mathbf{R}_{\text {rec,a }}$ can have eigenvalues which are orders of magnitude larger than the amplifier $T_{\min }$, so that the condition number (77) is large. To interpret the meaning of these large eigenvalues physically, we must return to the beam equivalent temperature as defined in (38). Since there is a limit on the maximum noise power that can be contributed by the amplifier (referred to the receiver outputs rather than to equivalent noise power at the source), large eigenvalues must correspond to small values of the denominator of (38). For a lossless array, from (11) we have $\mathbf{R}_{\mathrm{t}}=\mathbf{R}_{\text {iso }}$, from which it can be seen that the corresponding eigenvectors are beamformers that receive very little power due to an isotropic external thermal noise distribution. An equivalent passive antenna with the same receiving characteristics as the beamformer has a low radiation resistance and a poor match between the antenna terminals and load. Receiving a small isotropic noise power is also characteristic of receive superdirectivity, since directivity for a receive array can be formulated with the isotropic noise power received by the array in the denominator [21]. This is also reflected in the geometrical quality 
factor or superdirectivity measure $[28,29]$

$$
Q_{g}=\frac{\mathbf{w}^{H} \mathbf{w}}{\mathbf{w}^{H} \tilde{\mathbf{R}}_{\mathrm{iso}} \mathbf{w}}
$$

where $\tilde{\mathbf{R}}_{\text {iso }}$ is normalized to have unit diagonal elements as in (54). For a lossy array, $\mathbf{R}_{\mathrm{t}}$ includes a contribution from antenna element thermal noise which regularizes the matrix and limits the largest possible beam equivalent receiver noise temperature.

\section{NUMERICAL RESUltS}

As a simple and readily reproducible test case, we will use a numerical model to study the noise penalty due to mutual coupling for a linear array of parallel dipoles with several different amplifier noise matching conditions. To model amplifier noise using (38) or (49), the array mutual impedance matrix or scattering matrix is needed. The mutual impedances are approximated analytically using the induced EMF method [30]. Since a dipole is close to minimum scattering with an open circuit load, as discussed in Section II the open circuit loaded embedded element patterns in (1) can be approximated by the isolated element patterns, which for dipoles are available analytically. The element patterns are loaded using (2) to obtain the receiver output voltages $\mathbf{d}_{\mathrm{sig}}=\mathbf{Q} \mathbf{v}_{\mathrm{sig}, \mathrm{oc}}$ for a plane wave with a given angle of incidence $\Omega_{\text {sig }}$.

This procedure provides a signal steering vector which can be used to compute beamformer weights. We use the conjugate field match (CFM) beamformer

$$
\mathbf{w}=\mathbf{d}_{\mathrm{sig}}
$$

For array spacings larger than roughly one half wavelength, this beamformer achieves close to optimal gain. This beamformer does not maximize the beam sensitivity or $G / T$, since the beamformer does not take into account the noise correlation matrix. Using the CFM beamformer simplifies the treatment considerably, since the optimum $G / T$ beamformer weights depend on the receiver noise correlation matrix, which for the active and multi-beam optimal noise matching condition in turn depends on the beamformer weights. We will defer the development of a strategy for dealing with this circular dependence to future work.

Front end amplifier noise is included in the system model using (22). The amplifier input impedances are $\mathbf{Z}_{\mathrm{R}}=Z_{0} \mathbf{I}$ and the minimum amplifier noise temperature is $T_{\min }=20 \mathrm{~K}$. Noise matching is accomplished using the approach of Section III-A to adjust the optimal reflection coefficient $\Gamma_{\text {opt }}$ in (30) for each amplifier while maintaining fixed values for the noise resistance $R_{n}$ and minimum noise temperature $T_{\min }$.

\section{A. Element Spacing}

We will first characterize the degree of mutual coupling of the array as a function of element spacing using an intrinsic, beamformer- and amplifier-independent measure of the degree of mutual coupling. For this purpose, we employ the average element efficiency [31]

$$
\eta_{\mathrm{el}, \mathrm{avg}}=1-\frac{1}{M} \operatorname{trace}\left(\mathbf{S}_{A} \mathbf{S}_{A}^{H}\right)
$$

This measure of mutual coupling includes mismatch at each element port due to the diagonal S-parameters $S_{A m, m}$. To remove the contribution due to mismatches and measure only the mutual coupling, in this expression only we compute the S-parameters relative to the self impedances at each port instead of the system impedance $Z_{0}=50 \Omega$. Figure 2 shows the self impedance matched average element efficiency for two element and five element arrays.

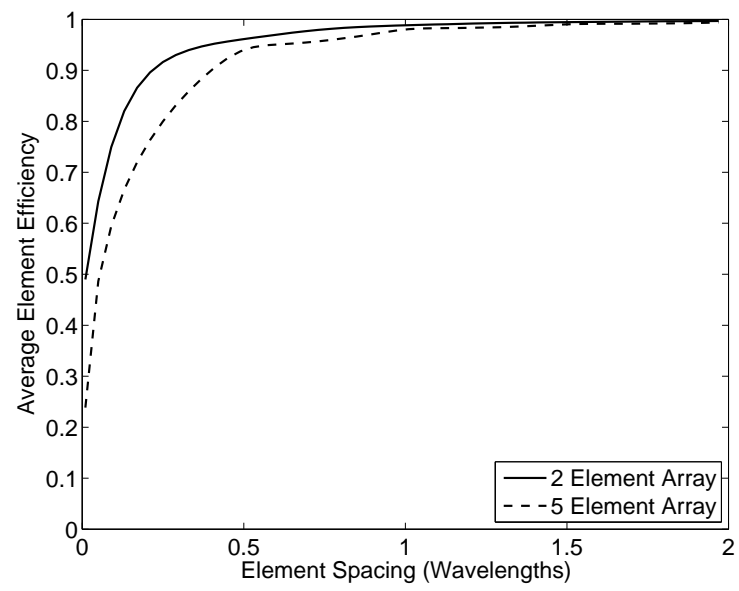

Fig. 2. Self-impedance matched average element efficiency as a function of element spacing for two and five element arrays. The average element efficiency is an intrinsic measure of the degree of mutual coupling.

We now consider the noise performance as a function of element spacing. The noise matching efficiency (40) is shown for two and five element arrays in Figures 3 and 4. In both cases, the amplifiers are matched to the self impedances of the array elements according to (69). The beam is steered to the broadside direction. As expected, the noise matching efficiency decreases as the spacing becomes small and the array becomes more strongly coupled. The deviation of the effective amplifier noise temperature from the optimal value increases as the noise resistance becomes larger. Noise matching efficiencies are somewhat smaller for the five element array than for the two element array. As the number of elements in the array increases, the noise matching efficiency for the linear array studied here tends to a constant, so that for large arrays, the noise matching efficiency is independent of the number of elements. 


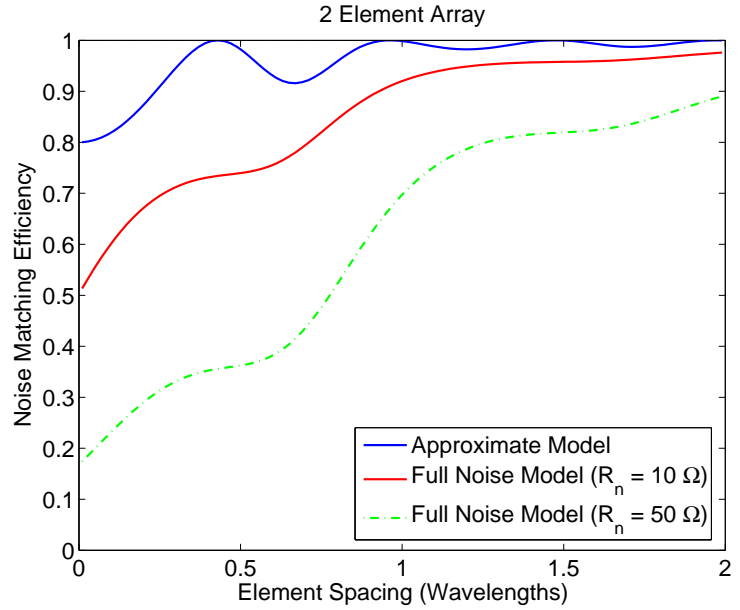

Fig. 3. Noise matching efficiency as a function of element spacing for a two element dipole array with the self impedance matching condition. For the approximate model (52), the array mutual impedance is approximated as purely real and the amplifiers are conjugate matched to the array. The noise matching efficiency becomes poorer with increasing amplifier noise resistance $R_{n}$ and decreasing element spacing.

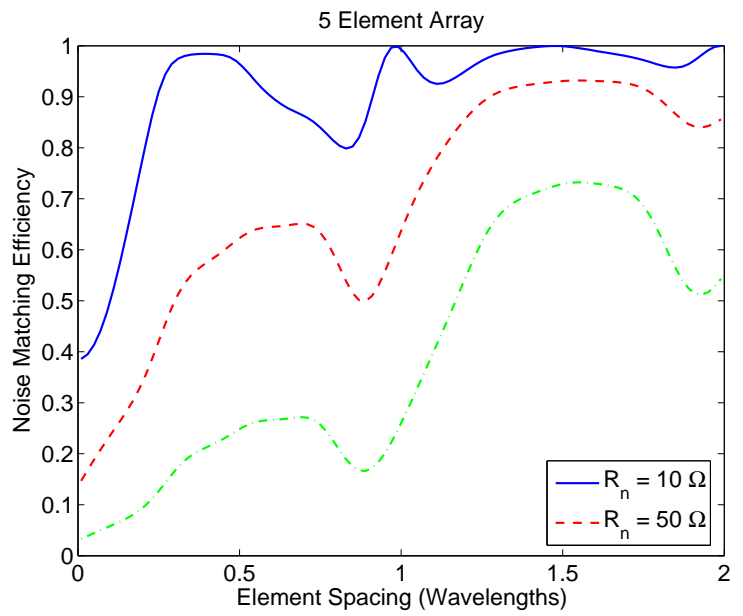

Fig. 4. Noise matching efficiency as a function of element spacing for a five element array.

\section{B. Beam Steering Direction}

We now consider the variation of the noise penalty for an array with fixed element spacing $(0.4 \lambda)$ with respect to the beam steering direction. For these results, $R_{n}=50 \Omega$, and the active impedance match is computed for the angle $0^{\circ}$ or broadside. Figure 5 shows the beam equivalent receiver noise temperature as a function of steering angle away from boresight for a twoelement array. Figure 6 shows the corresponding noise matching efficiency. Since the active impedance matching condition depends on the beamformer coefficients, the matching condition must be designed for a given beamformer. For the active impedance match, the noise matching efficiency is unity at $0^{\circ}$, as expected, but the efficiency decreases as the steering angle moves away from broadside. For the self impedance and average optimal matching conditions, the noise matching efficiency is never unity, but the sensitivity to steering angle is small.

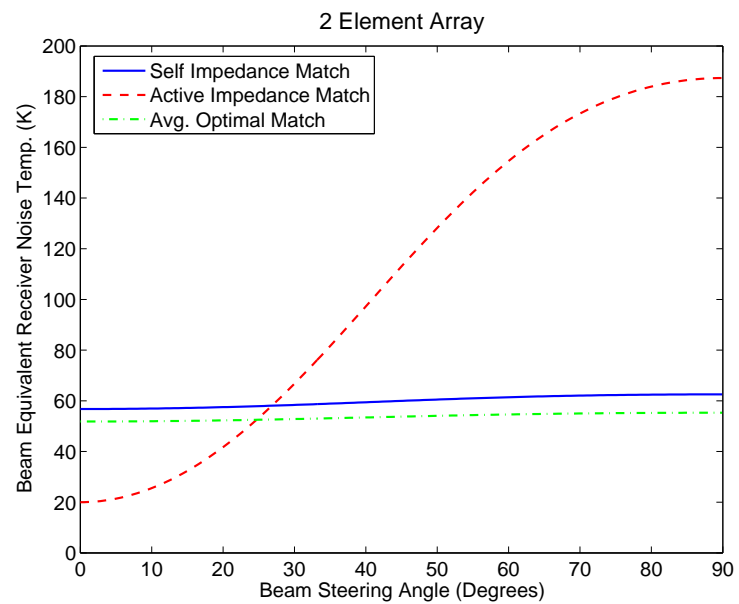

Fig. 5. Beam equivalent receiver noise temperature as a function of beam steering angle for a two element dipole array for the self impedance noise matching condition, the active impedance match at broadside $\left(0^{\circ}\right)$, and the multi-beam average optimal match given by Equation (73).

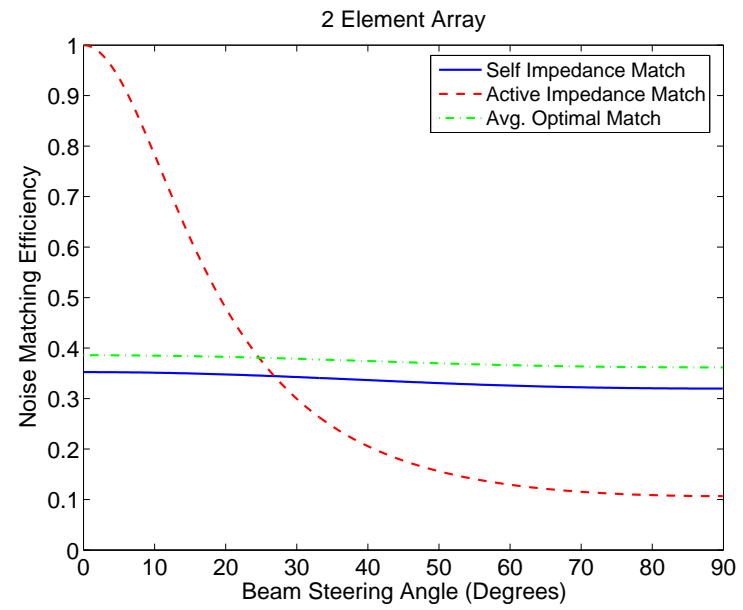

Fig. 6. Noise matching efficiency as a function of beam steering angle for a two element dipole array.

Figure 7 is a Smith chart representation of the active reflection coefficients defined in (62) as a function of beam steering angle, along with markers for the matched active reflection coefficient, amplifier $\Gamma_{\text {opt }}$ values for the multi-beam average optimal matching condition, and the self impedances. Due to the symmetry of the array, the active reflection coefficients at broadside and the self impedances are the same for both elements. 
Figures 8, 9 and 10 show the beam equivalent receiver noise temperature, noise matching efficiency, and Smith chart representation of the noise matching conditions for a five element array. It can be seen that the deviation of the receiver noise for the active impedance matching condition from $T_{\min }$ is much more severe for the larger array.

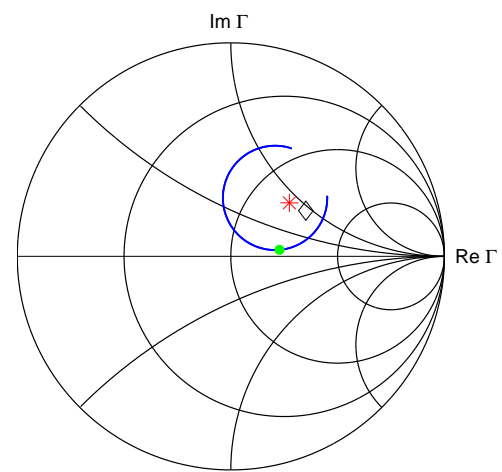

Fig. 7. Active reflection coefficients as a function of beam steering angle for the two element array. Markers show the active reflection coefficient at broadside $(\bullet)$, self impedances $(*)$, and multi-beam average optimal impedances $(\diamond)$. By symmetry, the self and active impedances are identical for the two elements.

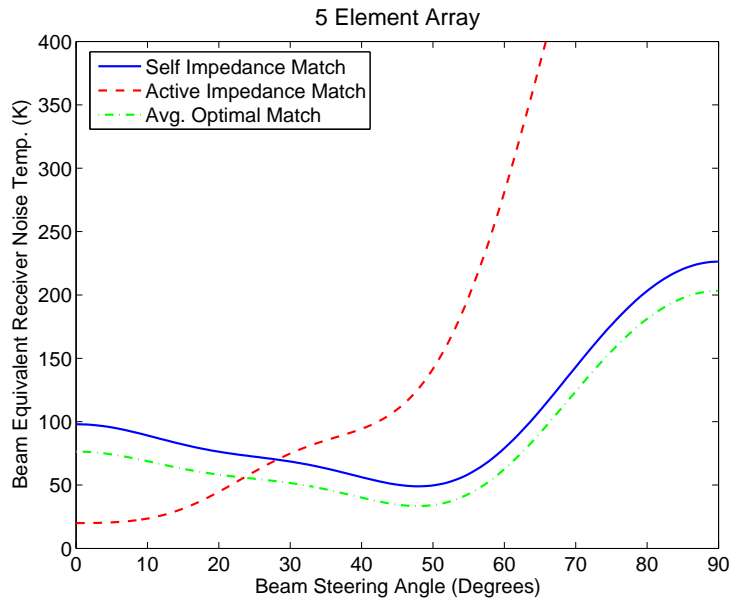

Fig. 8. Beam equivalent receiver noise temperature as a function of beam steering angle for a five element dipole array. The matching conditions are as in Fig. 5.

\section{Available Receiver Noise Correlation Matrix Spec- trum}

The noise matching efficiency results shown above can be interpreted in terms of the spectral properties of the available receiver noise temperature correlation matrix $\mathbf{R}_{\text {rec,a }}$ defined in (75). In Figures 11 and 12, the maximum and minimum eigenvalues are shown for two and five element arrays for the various noise

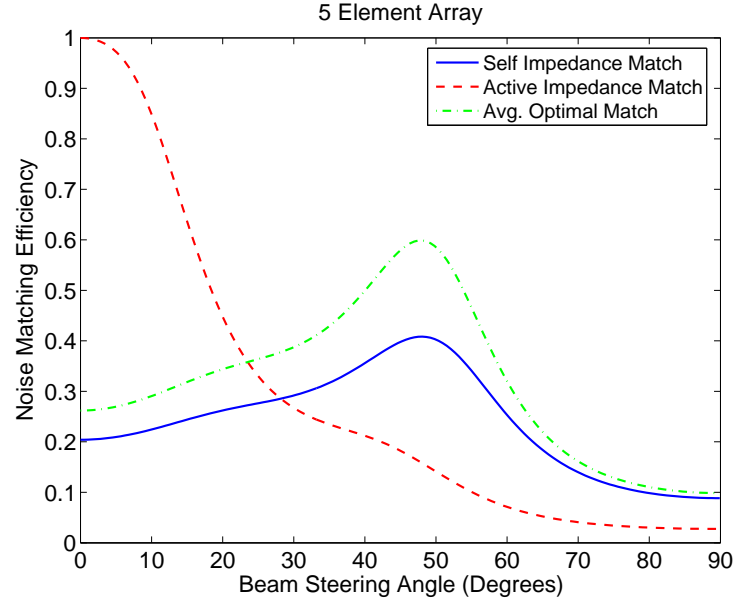

Fig. 9. Noise matching efficiency as a function of beam steering angle for a five element dipole array. The matching conditions are as in Fig. 5.

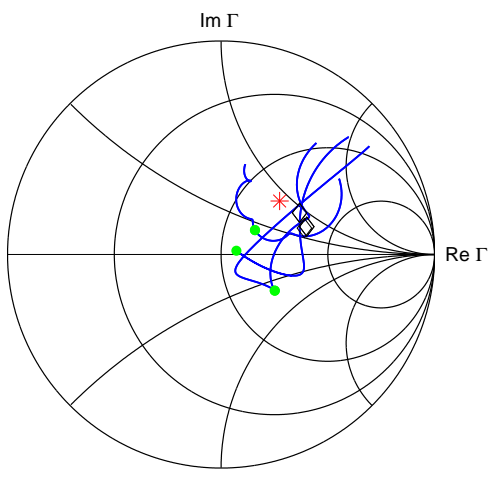

Fig. 10. Active reflection coefficients as a function of beam steering angle for the five element array. Markers are as in Fig. 7.

matching conditions. The smallest eigenvalue determines the smallest achievable beam equivalent receiver noise temperature, and the largest eigenvalue is the worst case noise temperature over all possible beamformers. For small element spacings, the array becomes more strongly coupled, and the maximum eigenvalue increases. For the active impedance match, one of the eigenvalues of $\mathbf{R}_{\text {rec,a }}$ is equal to the minimum amplifier noise temperature $T_{\min }$. For the beamformer used to compute the active impedance, the beam equivalent receiver noise temperature is $T_{\min }$ and the noise matching efficiency is unity for that beamformer. For other beamformers, the noise temperature increases and the noise matching efficiency is less than unity.

In Figure 13 and 14, the inverse condition number of $\mathbf{R}_{\text {rec,a }}$ for two and five element arrays is shown. For a Hermitian, positive definite matrix such as $\mathbf{R}_{\text {rec,a }}$, the inverse condition number is equal to the ratio of the smallest and largest eigenvalues. This provides a measure of the eigenvalue spread, which determines the 


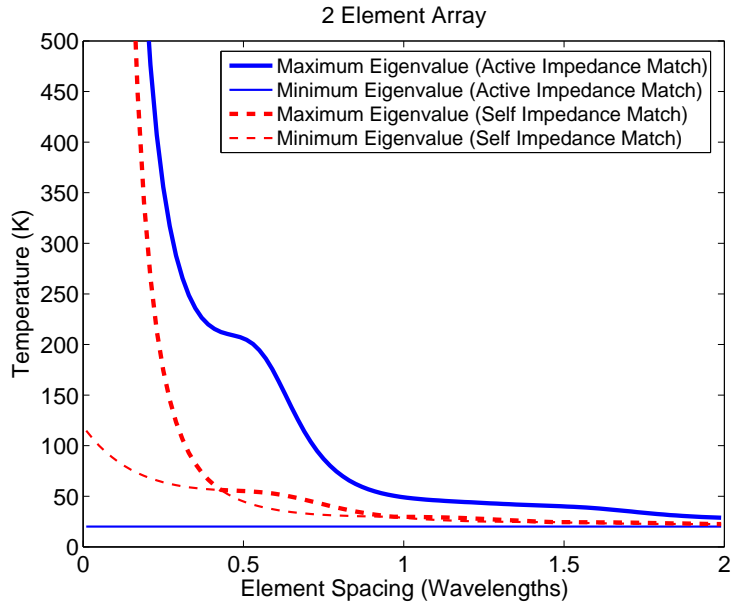

Fig. 11. Maximum and minimum eigenvalues of the available receiver noise temperature correlation matrix for a two element array.

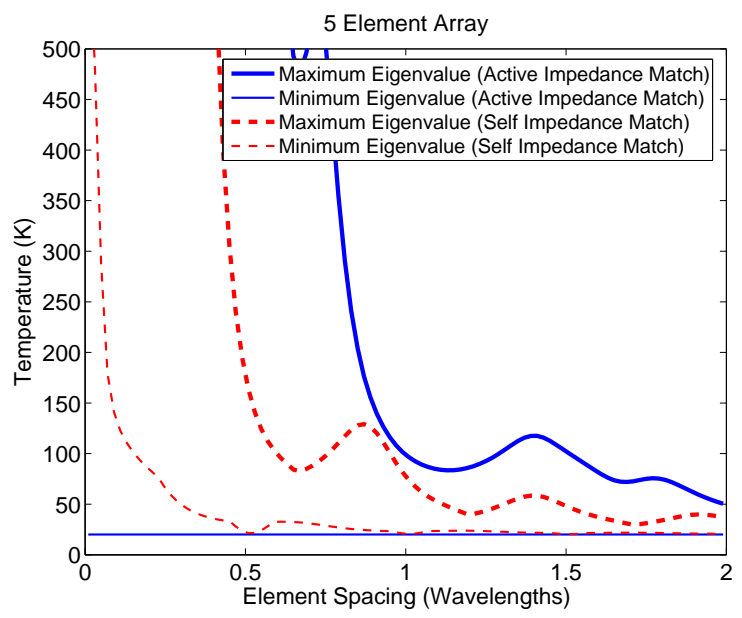

Fig. 12. Maximum and minimum eigenvalues of the available receiver noise temperature correlation matrix for a five element array.

sensitivity of the receiver noise temperature to the beam steering direction. It can be seen that the conditioning of the active impedance match is poorer than that of the self impedance match, but the difference decreases as the number of array elements increases.

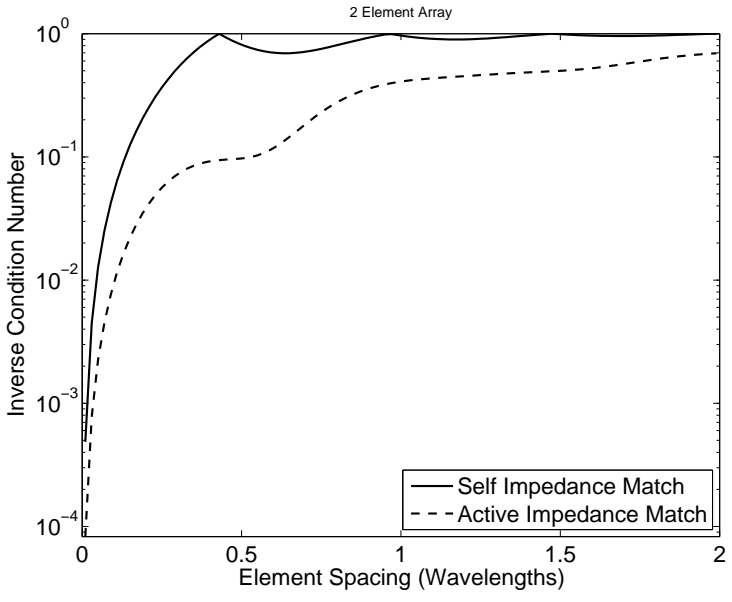

Fig. 13. Inverse condition number of the available receiver noise temperature correlation matrix for a two element array.

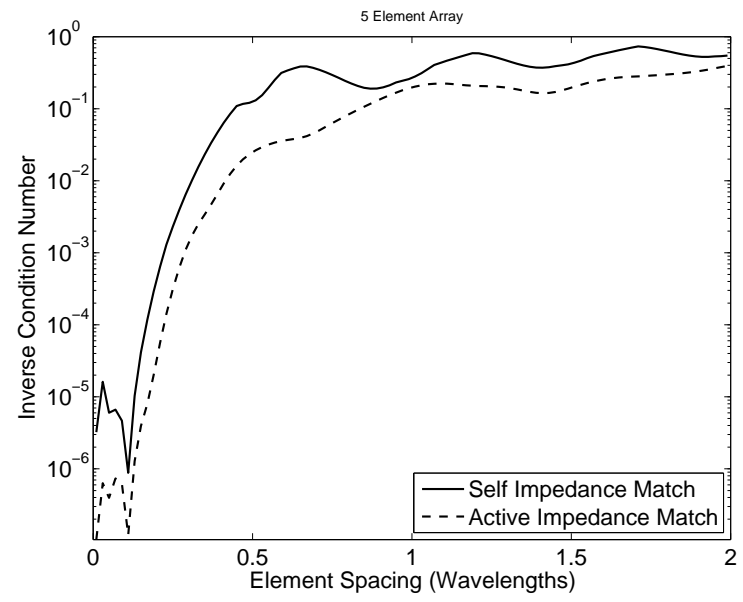

Fig. 14. Inverse condition number of the available receiver noise temperature correlation matrix for a five element array. For very small element spacings, numerical error is apparent. 


\section{CONCLUSIONS}

This paper has given an analysis of receiver noise for a mutually coupled beamforming array. The effects of mutual coupling on the signal and external noise response of an array can in principle be removed in signal processing, but amplifier noise depends on the source impedance in a way that cannot be compensated for in signal processing. For passive antennas, by convention receiver noise is referred to available power at the antenna terminals, since minimum available receiver noise implies maximum beam output SNR. For an active array, this concept generalizes to an available receiver noise temperature correlation matrix. The eigenvalues of this matrix have units of temperature, and the spectrum defines the range of possible beam equivalent receiver noise temperatures. The spectrum depends on the impedance seen by the amplifiers looking towards the array element ports, and noise matching conditions can be compared using the spectral properties of the available receiver noise temperature correlation matrix. For a given beamformer, the beam noise matching efficiency can be defined as the ratio of the beam equivalent noise temperature to the noise temperature of one front end amplifier and receiver chain under ideal noise matching conditions.

Using the noise matching efficiency and available receiver noise correlation matrix, we have compared the noise performance of the self and active impedance amplifier noise matching conditions to a multi-beam average optimal noise matching condition. The active matching condition achieves an optimal receiver noise temperature for a given beam steering direction, whereas the multi-beam matching condition achieves the minimum average receiver noise over a range of beam steering directions. For the array configurations considered in this paper, numerical results show that the self impedance and average optimal matching conditions are better conditioned, in the sense that the worst case noise temperature and sensitivity to the steering direction are smaller than for the active impedance match. The results also underscore the importance of front end amplifiers with low noise resistance. The amplifier noise resistance determines the sensitivity of the receiver noise to changes in the beamformer coefficients, so for applications with low noise or high stability requirements, minimizing the amplifier noise resistance is essential.

It is hoped that these results will help to solve open questions in the optimal noise matching of arrays for MIMO communications and low noise, high sensitivity applications such as radio astronomy and remote sensing. The challenge is determining the best amplifier noise matching condition, taking into account design factors such as the amplifier noise resistance, ohmic losses in noise matching networks, the mutual coupling properties of the array, and the space of beams to be formed with the array. Using the beam equivalent noise temperature or the eigenvalues of the available receiver noise temperature correlation matrix for different matching conditions, the goodness of noise matching conditions can be compared quantitatively and the best matching condition identified.

\section{ACKNOWLEDGEMENT}

This work has been carried out during a research stay of Karl Warnick at the Technische Universität München supported by the Deutscher Akademischer Austauschdienst (DAAD).

\section{REFERENCES}

[1] P. W. Hannan, "The element-gain paradox for a phased-array antenna," IEEE Trans. Ant.Propag., vol. 12, pp. 423-433, Jul. 1964.

[2] W. P. Geren, C. R. Curry, and J. Andersen, "A practical technique for designing multiport coupling networks," IEEE Trans. Microw. Th. Tech., vol. 44, pp. 364-371, Mar. 1996.

[3] S. Dossche, S. Blanch, and J. Romeu, "Three different ways to decorrelate two closely spaced monopoles for MIMO applications," in Proceedings of IEEE/ACES International Conference on Wireless Communications and Applied Computational Electromagnetics, pp. 849-852, Apr. 2005.

[4] S. Dossche, J. Rodriguez, S. Blanch, and J. Romeu, "Decoupling of a two-element switched dual band path antenna for optimum MIMO capacity," in Proceedings of IEEE Antennas and Propagation Society International Symposium, Jul. 2006.

[5] M. Jensen and J. Wallace, "Analysis of coupling in multi-antenna communication systems," IEICE Trans., vol. E87-C, pp. 14181424, Sep. 2004.

[6] K. F. Warnick and M. A. Jensen, "Optimal noise matching for mutually-coupled arrays," IEEE Transactions on Antennas and Propagation, vol. 55, pp. 1726-1731, June 2007.

[7] K. F. Warnick and B. D. Jeffs, "Beam efficiencies and system temperature for a focal plane array," Tech. Rep. http://hdl.handle.net/1877/588, Brigham Young University, Provo, UT, Nov. 2007.

[8] K. F. Warnick and B. D. Jeffs, "Beam efficiencies and system temperature for a focal plane array," IEEE Ant. Wireless Propag. Lett., no. in review, 2007.

[9] H. J. Chaloupka and X. Wang, "On the properties of small arrays with closely spaced antenna elements," in Proc. IEEE International Antenna and Propagation Symposium, 2004.

[10] K. F. Warnick and M. A. Jensen, "Effect of mutual coupling on interference mitigation with a focal plane array," IEEE Trans. Ant. Propag., vol. 53, pp. 2490-2498, Aug. 2005.

[11] C. Craeye, B. Parvais, and X. Dardenne, "MoM simulation of signal-to-noise patterns in infinite and finite receiving antenna arrays," IEEE Trans. Ant. Propag., vol. 52, pp. 3245-3256, Dec. 2004.

[12] E.E.M. Woestenburg, "Noise matching in dense phased arrays," Tech. Rep. RP-083, ASTRON, Dwingeloo, The Netherlands, Aug. 2005.

[13] R. Maaskant and E.E.M. Woestenburg, "Applying the active antenna impedance to achieve noise match in receiving array antennas," in Proc. IEEE International Antenna and Propagation Symposium, June 2007.

[14] A. T. De Hoop, "The n-port receiving antenna and its equivalent electrical network," Phillips Research Reports, vol. 30, pp. 302$315,1975$.

[15] P. S. Kildal, "Equivalent circuits of receive antennas in signal processing arrays," Microwave and Optical Technology Letters, vol. 21, no. 4, pp. 244-246, 1999. 
[16] W. Kahn and H. Kurss, "Minimum scattering antennas," IEEE Trans. Ant. Propag., vol. 13, pp. 671-675, Sep. 1965.

[17] "Application of the minimum scattering antenna theory to mismatched antennas," IEEE Trans. Ant. Propag., vol. 34, pp. 1223 1228, Oct. 1986.

[18] W. B. Davenport and W. L. Root, An Introduction to the Theory of Random Signls and Noise. New York: McGraw-Hill, 1958.

[19] H. Hillbrand and P. Russer, "An efficient method for computer aided noise analysis of linear amplifier networks," IEEE Trans. Circuits Systems, vol. 23, pp. 235-238, Apr. 1976.

[20] P. Russer and S. Müller, "Noise analysis of linear microwave circuits," International Journal of Numerical Modelling, Electronic Networks, Devices and Fields, vol. 3, pp. 287-316, 1990.

[21] K. F. Warnick and B. D. Jeffs, "Gain and aperture efficiency for a reflector antenna with an array feed," IEEE Antennas and Wireless Propagation Letters, vol. 5, no. 1, pp. 499-502, 2006.

[22] R. Q. Twiss, "Nyquist's and Thevenin's theorems generalized for nonreciprocal linear networks," J. Applied Phys., vol. 26, pp. 599-602, May 1955.

[23] S. Stein, "On cross coupling in multiple-beam antennas," IRE Trans. Ant. Propag., vol. 10, pp. 548-557, Sep. 1962.

[24] J. Engberg and T. Larsen, Noise Theory of Linear and Nonlinear Circuits. John Wiley \& Sons, 1995.

[25] "IEEE standard definitions of terms for antennas." IEEE Std 1451993.

[26] H. Bosma, "On the theory of linear noisy systems," Philips Research Reports, Supplement, no. 10, pp. 1-189, 1967.

[27] S. W. Wedge and D. B. Rutledge, "Noise waves and passive linear multiports," IEEE Microw. Guided Wave Lett., vol. 1, pp. 117 119, May 1991.

[28] M. Uzsoky and L. Solymar, "Theory of super-directive linear arrays," Acta Phys. Acad. Sci. Hung., vol. 6, pp. 195-204, 1956.

[29] Y. T. Lo, S. W. Lee, and Q. H. Lee, "Optimization of directivity and signal-to-noise ratio of an arbitrary antenna array," Proc. IEEE, vol. 54, pp. 1033-1045, Aug. 1966.

[30] C. A. Balanis, Advanced Engineering Electromagnetics. New York: John Wiley \& Sons, 1989.

[31] W. Kahn, "Element efficiency: a unifying concept for array antennas," IEEE Antennas Propag. Mag., vol. 49, pp. 48-56, Aug. 2007. 